\title{
Complex Riemannian Geometry-Bianchi Identities and Einstein Tensor
}

\author{
Richard A. Hutchin \\ Optical Physics Company, Calabasas, CA, USA \\ Email: rahutchin@opci.com
}

Received 28 July 2015; accepted 18 September 2015; published 21 September 2015

Copyright (C 2015 by author and Scientific Research Publishing Inc.

This work is licensed under the Creative Commons Attribution International License (CC BY). http://creativecommons.org/licenses/by/4.0/

(c) $\underset{\mathrm{EY}}{\mathrm{C}}$ Open Access

\begin{abstract}
Riemannian geometry has proved itself to be a useful model of the gravitational phenomena in the universe, but generalizations of it to include other forces have so far not been successful. Here we explore an extension of Riemannian geometry using a complex Hermitian metric tensor. We find that the standard electromagnetic field naturally appears along with two additional fields, which act as mass and charge sources. A first paper set up the basic geometry and derived the Christoffel symbols plus the E\&M field equation. This paper continues development with the generalized Riemann curvature tensor, Bianchi identities and the Einstein tensor, laying the basis for field equations. A final paper will then present the field equations.
\end{abstract}

\section{Keywords}

Unified Field Theory, Gravity, Electromagnetism, Complex Riemannian Geometry, Riemannian Geometry

\section{Introduction}

There have been numerous attempts to embed electromagnetism into Riemannian geometry, of which the most well known are Weyl's gauge field [1], Kaluza's fifth dimension [2] and Eddington's affine geometry [3]. Even exploration of making the metric tensor complex (as we propose here) was investigated almost a hundred years ago by Mie [4] and Reichenbacher [5]. These two did not have general relativity available at that time, and their theories led nowhere.

We explore here the implications of assuming a complex Riemannian geometry as a generalized theory of gravity. We find that the electromagnetic field naturally appears as the imaginary part of the metric tensor and that two other fields also emerge, denoted as $S$ and $W$. The flow of the development has 5 steps listed below. The first paper [6] set up the complex geometry and completed Step 1. This paper completes Steps 2-4, and a 
third paper will explore the field equations.

1) Derivation of the Christoffel symbols leading to the E\&M field equation;

2) Derivation of the Riemann curvature tensors;

3) Symmetries of the curvature tensors including Bianchi identities;

4) Derivation of the Einstein tensor;

5) Field equations for all four fields.

\section{Short Summary of the First Paper}

We provide here a summary of the first paper [6], which assumed a complex Riemannian geometry as a generalized theory of gravity. We found that the electromagnetic field naturally appeared as the imaginary part of the metric tensor and that two other fields also emerge, denoted as $S$ and $W$. That paper presented the concepts of complex Riemannian geometry and derives the Christoffel symbols, the E\&M field equation and the two additional fields. While the overall geometry is based on complex numbers, each of the four fields is pure real. The current paper derives the Riemann curvature tensor, Bianchi identities and the generalized Einstein tensor which can be used to provide field equations.

Since general relativity has been a successful theory of gravity, any generalization should reduce to the standard theory in certain approximations. Hence in exploring complex Riemannian geometry, there is strong motivation to include an axiomatic structure which closely parallels the familiar Riemannian geometry. A reference text on General Relativity will be useful to some readers to fill in details of the steps, for the structure of that formulation applies in broad terms. An example of a suitable text would be Introduction to General Relativity by Adler, Bazin and Schiffer (ABS) [7], but there are many available.

We began with a complex Hermitian metric tensor $c_{\alpha \beta}$ which defined an inner product between covariant complex vectors. The barred index is meant to indicate a complex-conjugate index, so that the inner product of a vector with itself will be real as defined in Equation (2.1), where ${ }^{*}$ indicates complex conjugation.

$$
|A|^{2}=A^{\alpha} C_{\alpha \bar{\beta}}\left(A^{\beta}\right)^{*}
$$

This form of $c_{\alpha \bar{\beta}}$ can be written as a sum of its real and imaginary parts as shown in Equation (2.2a) with its Hermitian inverse $c^{\bar{\beta} \alpha}$ also decomposed into real and imaginary parts as shown in Equation (2.2b). We note that since the two indices are typed for the complex metric tensors $c_{\alpha \bar{\beta}}$ and $c^{\bar{\beta} \alpha}$, the indices can be in any order without confusion such as $c_{\bar{\beta} \alpha}$ and $c^{\alpha \bar{\beta}}$-a convention that will simplify some of the later equations. We will also use the same convention for $f_{\alpha \bar{\beta}}=f_{\bar{\beta} \alpha}$ and its raised form $F^{\bar{\beta} \alpha}=F^{\alpha \bar{\beta}}$. Since the real part of $c_{\bar{\beta} \alpha}$ and $c^{\alpha \bar{\beta}}$ are symmetric, typing is not required for those two $\left(\boldsymbol{g}_{\alpha \beta}\right.$ and $\left.G^{\beta \alpha}\right)$.

$$
\begin{gathered}
c_{\alpha \bar{\beta}}=\boldsymbol{g}_{\alpha \beta}+i \boldsymbol{f}_{\alpha \bar{\beta}} \\
c^{\bar{\beta} \alpha}=G^{\beta \alpha}-i F^{\bar{\beta} \alpha}
\end{gathered}
$$

Here $\boldsymbol{g}$ is the usual symmetric metric tensor and $\boldsymbol{f}$ is an antisymmetric tensor. We will use bold font for those two basic fields, ( $\boldsymbol{g}_{\alpha \beta}$ and $\boldsymbol{f}_{\alpha \beta}$ ) as well as the two others ( $\boldsymbol{S}$ and $\boldsymbol{W}$ ), which naturally appear later. Thus it will be easy for the reader to identify the key fields. One may correctly surmise from this notation that $f$ will be identified with electromagnetism. Since $G^{\beta \alpha}$ is not the inverse of $\boldsymbol{g}_{\alpha \beta}$ due to the impact of the imaginary part $\boldsymbol{f}_{\alpha \beta}$, we will use the standard notation $\boldsymbol{g}^{\beta \alpha}$ for the inverse of $\boldsymbol{g}_{\alpha \beta}$. With this definition, we list some algebraic relationships in Equations (2.2c)-(2.2g). By expanding the equation $c^{\bar{\alpha} \beta} c_{\beta \bar{\gamma}}=c_{\gamma \bar{\beta}} c^{\bar{\beta} \alpha}=\delta^{\alpha}{ }_{\gamma}$ into real and imaginary parts, we can derive (2.2c)-(2.2e). Multiply Equation (2.2c) by $\boldsymbol{g}^{\gamma \beta}$ to get Equation (2.2f) and likewise multiply Equation (2.2d) by $\boldsymbol{g}^{\gamma \beta}$ to get Equation (2.2g).

Equation (2.2f) shows that $G^{\bar{\alpha} \beta}$ equals $g^{\alpha \beta}$ except for terms second order and higher in $\boldsymbol{f}_{\alpha \bar{\beta}}$. When we limit our consideration to second order terms, $G^{\bar{\alpha} \beta}$ can be replaced by $\boldsymbol{g}^{\alpha \beta}$.

Using this result, Equation (2.2g) shows that $F^{\bar{\alpha} \beta}$ equals $\boldsymbol{g}^{\alpha \mu} \boldsymbol{f}_{\mu \bar{\nu}} \boldsymbol{g}^{\nu \beta}$ except for terms third order and higher in $\boldsymbol{f}_{\alpha \beta}$. Thus when we limit our consideration to second order terms, $F^{\bar{\alpha} \beta}$ can be replaced by the familiar raised form of the E\&M tensor, $\boldsymbol{f}^{\alpha \beta}$.

$$
\begin{gathered}
G^{\bar{\alpha} \mu} \boldsymbol{g}_{\mu \gamma}+F^{\bar{\alpha} \mu} \boldsymbol{f}_{\mu \gamma}=\boldsymbol{g}_{\gamma \mu} G^{\bar{\mu} \alpha}+\boldsymbol{f}_{\gamma \mu} F^{\bar{\mu} \alpha}=\delta^{\alpha}{ }_{\gamma} \\
F^{\bar{\alpha} \mu} \boldsymbol{g}_{\mu \gamma}=G^{\bar{\alpha} \mu} \boldsymbol{f}_{\mu \gamma}
\end{gathered}
$$




$$
\begin{gathered}
\boldsymbol{g}_{\alpha \mu} F^{\bar{\mu} \gamma}=\boldsymbol{f}_{\alpha \mu} G^{\bar{\mu} \gamma} \\
G^{\bar{\alpha} \beta}=\boldsymbol{g}^{\bar{\alpha} \beta}-F^{\bar{\alpha} \mu} \boldsymbol{f}_{\mu \bar{\tau}} g^{\tau \beta}=\boldsymbol{g}^{\alpha \beta}-\boldsymbol{g}^{\alpha \mu} \boldsymbol{f}_{\mu \bar{\nu}} G^{\bar{\nu} \rho} \boldsymbol{f}_{\rho \bar{\tau}} \boldsymbol{g}^{\tau \beta} \\
F^{\bar{\alpha} \beta}=\boldsymbol{g}^{\alpha \mu} \boldsymbol{f}_{\mu \bar{\nu}} G^{\bar{\nu} \beta}=G^{\bar{\alpha} \mu} \boldsymbol{f}_{\mu \bar{\nu}} \boldsymbol{g}^{\nu \beta}
\end{gathered}
$$

Raising and Lowering Indices: The metric tensor is customarily used for raising and lowering indices of vectors and tensors, and this property also applies to the Hermitian metric with one caveat- the conjugate quality of the index switches. Thus $c_{\alpha \bar{\beta}}$ applied to $A^{\alpha}$ gives $A_{\bar{\beta}}$ and vice versa as summarized in Equations (2.3)-(4). One implication is that barred indices must be contracted with barred indices, and unbarred with unbarred. We identify $c^{\bar{\beta} \alpha}$ as the matrix inverse of $c_{\alpha \bar{\beta}}$, one being used for raising indices and the other for lowering. This definition makes raising and lowering indices inverse operations as usual.

$$
\begin{aligned}
A_{\bar{\beta}} & =A^{\alpha} c_{\alpha \bar{\beta}} \\
A^{\alpha} & =A_{\bar{\beta}} c^{\bar{\beta} \alpha}
\end{aligned}
$$

We also assume that one can convert between barred and unbarred indices by complex conjugation, i.e. you can create a new tensor with indices of flipped type by taking the complex conjugation of a previous tensor.

$$
\begin{gathered}
A_{\bar{\beta}}=A_{\beta}{ }^{*} \\
A^{\alpha}{ }_{\bar{\beta}}=A^{\bar{\alpha}}{ }_{\beta}{ }^{*}
\end{gathered}
$$

\section{Covariant Derivative}

We begin with scalar differentiation. In classical relativity, the partial derivative and the covariant derivative of a scalar field $\phi$ are the same numerically and are denoted respectively as $\phi_{\mid \gamma}$ and $\phi_{\| \gamma}$, according to a familiar notation. We keep the same assumption here. Both types of scalar derivatives are numerically equal, as shown in Equation (3.1), but will differ on subsequent covariant derivatives according to their type.

$$
\phi_{\mid \gamma}=\phi_{\mid \bar{\gamma}}=\phi_{\mid \gamma}
$$

Vector Differentiation: Complex geometry also introduces covariant differentiation with Christoffel symbols in the standard fashion, where there are now two different types of indices for the vector and two for the derivative resulting in four different types of Christoffel symbols shown in Equations (3.2a)-(3.2d).

$$
\begin{aligned}
& A^{\alpha}{ }_{\| \gamma}=A^{\alpha}{ }_{1 \gamma}-\Gamma^{\alpha}{ }_{v \gamma} A^{v} \\
& A^{\alpha}{ }_{\| \bar{\gamma}}=A^{\alpha}{ }_{\mid \gamma}-\Gamma^{\alpha}{ }_{\bar{\gamma} \bar{\gamma}} A^{v} \\
& A^{\bar{\alpha}}{ }_{\| \gamma}=A^{\bar{\alpha}}{ }_{\mid \gamma}-\Gamma^{\bar{\alpha}}{ }_{\bar{\gamma} \gamma}^{\bar{y}} \\
& A^{\bar{\alpha}}{ }_{\| \bar{\gamma}}=A^{\bar{\alpha}}{ }_{\mid \gamma}-\Gamma_{\overline{\gamma \gamma}}^{\bar{\alpha}} A^{\bar{\gamma}}
\end{aligned}
$$

We note that the Christoffel symbols for the lowered indices can be derived from Equations (3.2a)-(3.2d) using $\phi=A^{v} A$, and Equation (3.1) for the covariant derivative of a scalar function-just as is frequently done in classical Riemannian geometry. The result from the first paper [6] is summarized below.

$$
\begin{aligned}
& B_{\alpha \| \gamma}=B_{\alpha \mid \gamma}+\Gamma_{\alpha \gamma}^{v} B_{v} \\
& B_{\alpha \| \bar{\gamma}}=B_{\alpha \mid \bar{\gamma}}+\Gamma_{\alpha \bar{\gamma}}^{v} B_{v} \\
& B_{\bar{\alpha} \| \gamma}=B_{\bar{\alpha} \mid \gamma}+\Gamma_{\bar{\alpha} \gamma}^{\bar{v}} B_{\bar{v}} \\
& B_{\bar{\alpha} \| \bar{\gamma}}=B_{\bar{\alpha} \mid \bar{\gamma}}+\Gamma_{\overline{\alpha \gamma}}^{\bar{\gamma}} B_{\bar{v}}
\end{aligned}
$$

Note that with all derivatives of a vector, the vector index is always contracted with one of the first two indic- 
es of the Christoffel symbol, which two indices are always of the same type (barred or unbarred). The last index is always the derivative index.

We also require that covariant differentiation maintain the conjugation symmetry. This last statement means that:

$$
\left(A^{\alpha}{ }_{\| \gamma}\right)^{*}=A^{\bar{\alpha}}{ }_{\| \bar{\gamma}}
$$

and

$$
\left(A^{\alpha}{ }_{\| \bar{\gamma}}\right)^{*}=A^{\bar{\alpha}}{ }_{\| \gamma} .
$$

Equations (3.4a) and (3.4b) imply conjugation symmetry on the Christoffel symbols, namely:

$$
\begin{aligned}
\left(\Gamma_{\rho \gamma}^{\alpha}\right)^{*} & =\Gamma_{\overline{\rho \gamma}}^{\bar{\alpha}} \\
\left(\Gamma^{\bar{\alpha}}{ }_{\bar{\rho} \gamma}\right)^{*} & =\Gamma_{\rho \bar{\gamma}}^{\alpha} .
\end{aligned}
$$

We will frequently need to use the explicit difference $S$ between the barred and unbarred Christoffel symbols and thus give it its own symbol as defined in Equation (3.6a) and (3.6b). Since the difference between two Christoffel symbols transforms as a tensor under coordinate transformations, we know that $\boldsymbol{S}$ must be a tensor, and we proved in the first paper [6] that $S$ is pure real. We also showed that $\boldsymbol{S}$ switches sign when the type of one of its indices switches, as it does in Equation (3.6).

$$
2 S_{\beta \gamma}^{\alpha}=2 \Gamma_{\beta \gamma}^{\alpha}-2 \Gamma_{\beta \bar{\gamma}}^{\alpha}=-2 S_{\beta \bar{\gamma}}^{\alpha}
$$

We note one immediate corollary to this definition of $\boldsymbol{S}$ in (3.7a) or equivalently in Equation (3.7b).

$$
\begin{aligned}
& A^{\alpha}{ }_{\| \gamma}-A^{\alpha}{ }_{\| \bar{\gamma}}=-\left(\Gamma_{\beta \gamma}^{\alpha}-\Gamma_{\beta \bar{\gamma}}^{\alpha}\right) A^{\beta}=-2 S_{\beta \gamma}^{\alpha} A^{\beta} \\
& A^{\bar{\alpha}}{ }_{\| \gamma}-A^{\bar{\alpha}}{ }_{\| \bar{\gamma}}=-\left(\Gamma_{\bar{\beta} \gamma}^{\bar{\alpha}}-\Gamma_{\overline{\beta \gamma}}^{\bar{\alpha}}\right) A^{\bar{\beta}}=-2 S^{\bar{\alpha}}{ }_{\bar{\beta} \gamma} A^{\bar{\beta}}
\end{aligned}
$$

Christoffel Symmetry Condition: Since we want this new geometry to reduce to classical Riemannian geometry when the metric tensor is pure real, we will want to impose symmetry conditions on the Christoffel symbols which map to the classical symmetry in the last two indices. We chose the condition shown in Equations (3.8a)-(3.8c), where the Christoffel is unchanged when the last two indices switch. As will be shown below, this condition leads to generalized Bianchi conditions and resulting Einstein tensor. An alternate symmetry, which used complex conjugation in Equation (3.8c), was also explored, but it was found to produce only a constant E\&M field—useless for this geometry.

$$
\begin{aligned}
& \Gamma_{\beta \gamma}^{\alpha}=\Gamma_{\gamma \beta}^{\alpha} \\
& \Gamma_{\overline{\beta \gamma}}^{\bar{\alpha}}=\Gamma_{\overline{\gamma \beta}}^{\bar{\alpha}} \\
& \Gamma_{\beta \bar{\gamma}}^{\alpha}=\Gamma_{\gamma \bar{\beta}}^{\alpha}
\end{aligned}
$$

Zero Derivatives for the Metric Tensor: The last requirement we want to make on the Christoffel symbols is that the covariant derivative of the metric tensor vanishes as usual. Since there are two types of covariant derivative, we get two equations - which are just complex conjugates of each other (with a flip of indices).

$$
\begin{gathered}
c_{\rho \bar{v} \| \gamma}=\Gamma_{\rho \gamma}^{\alpha} c_{\alpha \bar{\nu}}+c_{\rho \bar{\alpha}} \Gamma_{\bar{\nu}}^{\bar{\alpha}}+c_{\rho \bar{v} \mid \gamma}=0 \\
c_{\rho \bar{v} \| \bar{\gamma}}=\Gamma_{\rho \bar{\gamma}}^{\alpha} c_{\alpha \bar{\nu}}+c_{\rho \bar{\alpha}} \Gamma_{\bar{\nu}}^{\bar{\alpha}}+c_{\rho \bar{\nu} \mid \bar{\gamma}}=0
\end{gathered}
$$

By taking the average of these two equations, we note that there is a third derivative form in Equation (3.9c) that also gives a zero derivative for the metric tensor and thus may have a place in the geometry. Since this derivative is the average of barred and unbarred derivatives, we use a different slashed symbol. This form of derivative will be essential to derive the Riemann curvature tensor. All the forms of the barred, unbarred and slashed Christoffel symbols are summarized in Equation (3.13). 
We note as a caution that the derivation of the Christoffel symbols in the first paper [6] required the selection of a fully real Christoffel symbol to make the complex Christoffel symbols correctly invariant under coordinate transformations. We chose the classical Christoffel symbols for convenience and because they gave an elegant non-recursive formula for the Christoffel symbols. However, as a note for the future, we could also have chosen the fully slashed Christoffel symbols, which are also pure real and are more naturally embedded into this complex geometry. This would change some of the higher order terms in the field equations. Assuming this basic unification process is correct, then only experimental observations will be able to determine which path to take.

$$
\begin{gathered}
\Gamma_{b \psi}^{a}=\frac{1}{2}\left(\Gamma_{b \gamma}^{a}+\Gamma_{b \bar{\gamma}}^{a}\right) \\
c_{\rho \bar{v} \| \gamma}=\Gamma_{\rho \gamma}^{\alpha} c_{\alpha \bar{v}}+c_{\rho \bar{\alpha}} \Gamma_{\bar{v} \gamma}^{\bar{\alpha}}+c_{\rho \bar{v} \mid \gamma}=0
\end{gathered}
$$

The E\&M Field Equation:

Once we have the transpose symmetry for the Christoffel symbols (Equations (3.8)) and the zero derivative Equations (3.9), we can derive the desired E\&M field equation by antisymmetrizing Equation (3.9a) i.e. summing over all signed permutations of $\rho, v$ and $\gamma$. (This is denoted $\{\cdots \cdots\}_{(\rho, v, \gamma)}$. ) Since the Christoffel symbols are all symmetric in the lower indices, all the Christoffel terms vanish, leaving only one term in (3.11).

$$
\left\{\Gamma_{\rho \gamma}^{\alpha} c_{\alpha \bar{v}}+c_{\rho \bar{\alpha}} \Gamma_{\bar{v} \gamma}^{\bar{\alpha}}+c_{\rho \bar{v} \mid \gamma}\right\}_{(\rho, v, \gamma)}=\left\{c_{\rho \bar{v} \mid \gamma}\right\}_{(\rho, v, \gamma)}=0
$$

Expanding that metric tensor in (3.11), we get further simplification in Equation (3.12) since the symmetric $g_{\rho \underline{v}}$ term drops out in the antisymmetrization. What is left is $i$ times the standard E\&M field equation.

$$
\left\{c_{\rho \bar{v} \mid \gamma}\right\}_{(\rho, \gamma, v)}=\left\{\boldsymbol{g}_{\rho \bar{v} \mid \gamma}+i \boldsymbol{f}_{\rho \bar{v} \mid \gamma}\right\}_{(\rho, \gamma, v)}=i\left\{\boldsymbol{f}_{\rho \bar{v} \mid \gamma}\right\}_{(\rho, \gamma, v)}=0
$$

We note that this field Equation (3.11) is well known to imply that the E\&M field tensor $f_{\rho v}$ can be expressed as the antisymmetric derivative of a vector potential $A_{\rho}$ as shown in Equation (3.12) [7]. Thus we could have this E\&M field Equation (3.11) satisfied simply by using Equation (3.12) instead of $f_{\rho \underline{v}}$. This is often done in modern E\&M calculations.

$$
\boldsymbol{f}_{\rho \bar{v}}=A_{\rho \mid v}-A_{v \mid \rho}
$$

As a note on the indexing convention used here: A Greek index carries a type, either barred or unbarred, while a roman index indicates a more general index of either type. This convention makes many of the equations much simpler. For instance, the general solution for a lowered Christoffel symbol is shown in Equation (3.13a), with the only condition being that indices a and b must have the opposite type. The bold green Christoffel symbols are the standard ones from classical general relativity, and the bold green covariant derivatives use the classical covariant derivatives. With the classical derivatives and the barred and unbarred derivatives, this complex Riemannian geometry has a total of three different types of derivatives - each of which has its purpose. It is fascinating that the classical Christoffel symbols evolve intrinsically in this complex geometry-as explained in the first paper [6].

The same expression for the raised Christoffel symbols is given in Equation (3.13b). Note that the barred or unbarred type of index a is used to determine the sign of the $f$ terms. In Equation (3.13a), if index a is barred, then that index position is barred in the two $f$ terms to give $i\left(f_{b \bar{a} \| c}+f_{c \bar{a} \| b}\right) / 3$. If a is unbarred, then these terms become $i\left(f_{\bar{b} a \mid c}+f_{\bar{c} a \| b}\right) / 3$, which is minus the previous term. In Equation (3.13b), the type of index a determines the type of index $d$, which then determines the sign of the $\mathrm{f}$ terms as in the previous sentence. Equations (3.13c), (3.13d) and (3.13e) summarize the slashed Christoffel symbols.

$$
\begin{gathered}
\Gamma_{a b c}=c_{a d} \Gamma_{b c}^{d}-S_{a b c}+i\left(f_{b a \| c}+f_{c a \| b}\right) / 3+i W_{a b c} \\
\Gamma_{b c}^{a}=\Gamma_{b c}^{a}+c^{a d} S_{d b c}+i c^{a d}\left(f_{b d \| c}+f_{c d \| b}\right) / 3+i c^{a d} W_{d b c} \\
\Gamma_{b c}^{a}=\Gamma_{b c}^{a}-i F^{a d} S_{d b c}+F^{a d}\left(f_{b d \| c}+f_{c d \| b}\right) / 3+F^{a d} W_{d b c} \\
\Gamma_{b c}^{a}=\Gamma_{b c}^{a}+i c^{a d}\left(f_{b d \| c}+f_{c d \| b}\right) / 3+i c^{a d} W_{d b c}
\end{gathered}
$$




$$
\Gamma_{b c}^{*}=\Gamma_{b c}^{a}+F^{a d}\left(f_{b d \| c}+f_{c d \| b}\right) / 3+F^{a d} W_{a b c}
$$

For easy reference we summarize the symmetries of the $S$ and $W$ tensors here, derived in the previous paper.

The $S$ tensor changes sign if the type of the first two indices flip (which must be opposite types) or the third index flips.

$$
S_{\bar{\alpha} \beta \bar{\gamma}}=-S_{\bar{\alpha} \beta \gamma}=-S_{\alpha \bar{\beta} \gamma}=S_{\alpha \overline{\beta \gamma}}
$$

If types are fixed, then $S$ is invariant under all 6 permutations of the index values.

$$
S_{\bar{\alpha} \beta \bar{\gamma}}=S_{\bar{\alpha} \bar{\beta}}=S_{\bar{\beta} \alpha \bar{\gamma}}=S_{\bar{\beta} \gamma \bar{\alpha}}=S_{\bar{\gamma} \alpha \bar{\beta}}=S_{\bar{\gamma} \beta \bar{\alpha}}
$$

The $\mathrm{W}$ tensor changes sign if the type of the first two indices switch types, but index type makes no difference to the sign in the last index for $W$.

$$
W_{\bar{\alpha} \beta \bar{\gamma}}=W_{\bar{\alpha} \beta \gamma}=-W_{\alpha \overline{\beta \gamma}}=-W_{\alpha \bar{\beta} \gamma}
$$

Just like $\boldsymbol{S}$, if types are fixed, then $\boldsymbol{W}$ is invariant under all 6 permutations of the index values

$$
W_{\bar{\alpha} \beta \bar{\gamma}}=W_{\bar{\alpha} \gamma \bar{\beta}}=W_{\bar{\beta} \alpha \bar{\gamma}}=W_{\bar{\beta} \gamma \bar{\alpha}}=W_{\bar{\gamma} \alpha \bar{\beta}}=W_{\bar{\gamma} \beta \bar{\alpha}}
$$

With these symmetries, we note that the sum of two Christoffel symbols which switch type in the first two indices is twice the classical Christoffel symbol.

\section{Scalar Curvature Tensor}

Now we can deduce some general properties of this type of geometry. We start with the scalar curvature tensor where a scalar function is twice differentiated. When both derivatives are of the same type, then derivatives commute as usual as shown in Equations (4.1a) and (4.1b). However, when they are of different types, then they don't commute in general as shown in Equation (4.1c). Note that since all derivatives of a scalar field are identical, we can write Equation (4.1c) using the slashed derivative, which is obviously a tensor equation since the difference between two Christoffel symbols is a tensor under coordinate transformations.

$$
\begin{gathered}
\Phi_{\|\beta\| \gamma}-\Phi_{\|\gamma\| \beta}=\left(\Gamma_{\beta \gamma}^{v}-\Gamma_{\gamma \beta}^{v}\right) \Phi_{\| v}=0 \\
\Phi_{\|\bar{\beta}\| \bar{\gamma}}-\Phi_{\|\bar{\gamma}\| \bar{\beta}}=\left(\Gamma_{\overline{\beta \gamma}}^{\bar{\nu}}-\Gamma_{\overline{\gamma \beta}}^{\bar{\nu}}\right) \Phi_{\| \bar{v}}=0 \\
\Phi_{\|\beta\| \bar{\gamma}}-\Phi_{\|\bar{\gamma}\| \beta}=\left(\Gamma_{\beta \bar{\gamma}}^{v} \Phi_{\| v}-\Gamma_{\bar{\gamma} \beta}^{\bar{v}} \Phi_{\| \bar{v}}\right)=\left(\Gamma_{\beta \bar{\gamma}}^{v}-\Gamma_{\bar{\gamma} \beta}^{\bar{v}}\right) \Phi_{\| \psi}=K_{\beta \bar{\gamma}}^{\psi} \Phi_{\| \psi}
\end{gathered}
$$

The general result is given in Equations (4.2a) and (4.2b), where for convenience in writing these equations we also define the scalar curvature tensor $K_{a b}^{m}=\Gamma_{a b}^{m}-\Gamma_{b a}^{m}$, which vanishes unless the indices a and b are different types. With the symmetry in the lower two indices of a Christoffel symbol and the complex conjugation symmetry when the types are switched, $K_{a b}^{m}$ is either identically 0 or pure imaginary, and thus can be treated always as pure imaginary_obviously vanishing in the classical limit where all fields are real.

$$
\Phi_{\|a\| b}-\Phi_{\|b\| a}=K_{a b}^{m} \Phi_{\| m}
$$

where

$$
K^{m}{ }_{a b}=\Gamma_{a b}^{m}-\Gamma_{b a}^{m} \text { (pure imaginary) }
$$

Since the slashed derivative has been established as a third valid type of covariant derivative with its own Christoffel symbols, we point out that indices $a$ and $b$ in Equation (4.2b) can be slashed as well as barred or unbarred. The slashed scalar curvature tensor is simply and intuitively related to the unslashed scalar curvature tensor as shown in Equation (4.3)

$$
K_{a b}^{m}=\Gamma_{a b}^{m}-\Gamma_{b a}^{m}=\frac{1}{2}\left(\Gamma_{a b}^{m}+\Gamma_{a \bar{b}}^{m}-\Gamma_{b a}^{m}-\Gamma_{\bar{b} a}^{\bar{m}}\right)=\frac{1}{2}\left(K_{a b}^{m}+K_{a \bar{b}}^{m}\right)
$$

\section{Riemann Curvature Tensor}

We next derive the Riemann curvature tensor and explore its symmetries. It is defined as usual by taking the an- 
tisymmetrized double derivative of a vector - but in this derivative the vector may be barred or unbarred and each derivative may be barred or unbarred thereby creating 8 different curvature tensors. While this may seem like a large number, the symmetries will reduce them to 3 types.

Derivatives of the Same Type: To make this process explicit, we start with the simplest curvature tensor derivation, where all indices are of the same type. Begin with the definition of covariant derivative of an arbitrary twice differentiable vector field $A^{\alpha}$ in Equation (5.1a), then differentiate again in (5.1b), reverse the order of differentiation in (5.1c) and subtract the two in (5.1d). The terms involving $A^{\beta}$ subtract away, and the coefficient of the $A^{\alpha}{ }_{\mid v}$ factor is designated the Riemann curvature tensor as usual. Its final equation summarized in (5.1f) looks identical to the standard expression for the Riemann curvature tensor in textbooks, although the Christoffel symbols here are now complex.

$$
\begin{aligned}
& A^{\alpha}{ }_{\| \gamma}=A^{\alpha}{ }_{\mid \gamma}-\Gamma_{\beta \gamma}^{\alpha} A^{\beta} \\
& A^{\alpha}{ }_{\| \gamma \mid \delta}=A^{\alpha}{ }_{|\gamma| \delta}-\Gamma_{\beta \gamma \mid \delta}^{\alpha} A^{\beta}-\Gamma_{\beta \gamma}^{\alpha} A_{\mid \delta}^{\beta}-\Gamma_{\beta \delta}^{\alpha} A^{\beta}{ }_{\mid \gamma}+\Gamma_{\gamma \delta}^{\nu} A_{\mid v}^{\alpha}+\Gamma_{\nu \delta}^{\alpha} \Gamma_{\beta \gamma}^{\nu} A^{\beta}-\Gamma_{\gamma \delta}^{\nu} \Gamma_{\beta \nu}^{\alpha} A^{\beta} \\
& A^{\alpha}{ }_{\|\delta\| \gamma \gamma}=A_{|\delta| \gamma}^{\alpha}-\Gamma_{\beta \delta \mid \gamma}^{\alpha} A^{\beta}-\Gamma_{\beta \delta}^{\alpha} A_{\mid \gamma}^{\beta}-\Gamma_{\beta \gamma}^{\alpha} A_{\mid \delta}^{\beta}+\Gamma_{\delta \gamma}^{v} A_{\mid v}^{\alpha}+\Gamma_{v \gamma}^{\alpha} \Gamma_{\beta \delta}^{\nu} A^{\beta}-\Gamma_{\delta \gamma}^{v} \Gamma_{\beta \nu}^{\alpha} A^{\beta} \\
& A_{\|\gamma\| \delta}^{\alpha}-A_{\|\delta\| \gamma}^{\alpha}=\left(-\Gamma_{\beta \gamma \mid \delta}^{\alpha}+\Gamma_{\beta \delta \mid \gamma}^{\alpha}-\Gamma_{\nu \gamma}^{\alpha} \Gamma_{\beta \delta}^{\nu}+\Gamma_{\nu \delta}^{\alpha} \Gamma_{\beta \gamma}^{\nu}\right) A^{\beta} \\
& A^{\alpha}{ }_{\|\gamma\| \delta}-A^{\alpha}{ }_{\|\delta\| \gamma}=R_{\beta \gamma \delta}^{\alpha} A^{\beta} \\
& R_{\beta \gamma \delta}^{\alpha}=-\Gamma_{\beta \gamma \mid \delta}^{\alpha}+\Gamma_{\beta \delta \mid \gamma}^{\alpha}-\Gamma_{\nu \gamma}^{\alpha} \Gamma_{\beta \delta}^{v}+\Gamma_{\nu \delta}^{\alpha} \Gamma_{\beta \gamma}^{\nu}
\end{aligned}
$$

We note that the type of index $\alpha$ had no impact on the structure of the curvature tensor derivation above. Thus the same equation for the Riemann curvature tensor will work if $\alpha$ is barred or unbarred (and always the same type as $\beta$ ). Also $\gamma$ and $\delta$ above can be any two indices of the same type and the algebra will work the same. Thus Equation (5.1f) applies to all index combinations where $\gamma$ and $\delta$ have the same type.

Derivatives of Different Types: The derivation with mixed types of derivatives begins the same way in Equations (5.2a)-(5.2c), but the difference in Equation (5.2d) has two extra terms, marked in yellow because there are two types of derivatives - one barred and one unbarred. We must keep track of those two terms when doing mixed derivatives.

$$
\begin{aligned}
& A^{\alpha}{ }_{\| \gamma}=A^{\alpha}{ }_{y \gamma}-\Gamma_{\beta \gamma}^{\alpha} A^{\beta} \\
& A^{\alpha}{ }_{\| \gamma \mid \bar{\delta}}=A^{\alpha}{ }_{|\gamma| \delta}-\Gamma_{\beta \gamma \mid \delta}^{\alpha} A^{\beta}-\Gamma_{\beta \gamma}^{\alpha} A_{\mid \delta}^{\beta}-\Gamma_{\beta \bar{\delta}}^{\alpha} A^{\beta}{ }_{\mid \gamma}-\Gamma_{\gamma \bar{\delta}}^{v} A^{\alpha}{ }_{\| v}+\Gamma_{\nu \bar{\delta}}^{\alpha} \Gamma_{\beta \gamma}^{v} A^{\beta} \\
& A^{\alpha}{ }_{\|\bar{\delta}\| \gamma}=A_{|\delta| \gamma}^{\alpha}-\Gamma_{\beta \bar{\delta} \mid \gamma}^{\alpha} A^{\beta}-\Gamma_{\beta \bar{\delta}}^{\alpha} A_{\mid \gamma}^{\beta}-\Gamma_{\beta \gamma}^{\alpha} A_{\mid \delta}^{\beta}-\Gamma_{\bar{\delta} \gamma}^{\bar{\nu}} A_{\| \bar{v}}^{\alpha}+\Gamma_{\gamma \gamma}^{\alpha} \Gamma_{\beta \bar{\delta}}^{\nu} A^{\beta}
\end{aligned}
$$

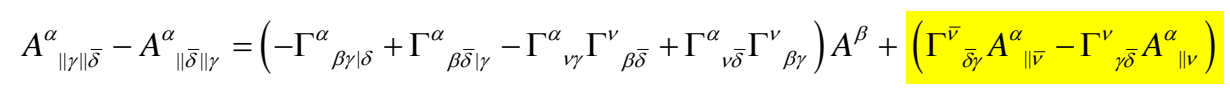

To find the curvature tensor we need to separate the right side of Equation (5.2d) into a tensor component multiplying $A^{\beta}$ and a tensor term multiplying the covariant derivative of $A^{\alpha}$. The part multiplying $A^{\beta}$ will then be the generalized Riemann curvature tensor.

To make this work, we need to use the slashed derivative as we did in Equation (4.1c), which is half way between the barred and unbarred derivatives. The algebra is shown in Equations (5.3a)-(5.3c). In Equation (5.3a) we expand the covariant derivatives. In Equation (5.3b), we add and subtract slashed Christoffel terms, which we regroup in Equation (5.3c). Finally we substitute that result into Equation (5.2d) to get Equation (5.3d), which has clearly separated out a covariant component (the same difference of Christoffel symbols we found in Equation (4.1c)) that multiplies the covariant derivative, leaving the rest to be the Riemann curvature tensor term.

For compactness we defined $\Delta \Gamma_{b c}^{a}=\Gamma_{b c}^{a}-\Gamma_{b c}^{a}$, which is itself a tensor under coordinate transformations since it is the difference between two Christoffel symbols. As shown in Equation (5.3e) (referencing Equation (3.6a)), it is exactly equal to the $S$ tensor $S^{a}{ }_{b c}$, which also equals $-S^{a}{ }_{b \bar{c}}$.

$$
\Gamma_{\bar{\delta} \gamma}^{\bar{\nu}} A_{\| \bar{v}}^{\alpha}-\Gamma_{\gamma \bar{\delta}}^{v} A_{\| v}^{\alpha}=\Gamma_{\bar{\delta} \gamma}^{\bar{V}}\left(A_{\mid v}^{\alpha}-\Gamma_{\beta \bar{v}}^{\alpha} A^{\beta}\right)-\Gamma_{\gamma \bar{\delta}}^{v}\left(A_{\mid v}^{\alpha}-\Gamma_{\beta \nu}^{\alpha} A^{\beta}\right)
$$




$$
\begin{aligned}
& \Gamma_{\overline{\delta \gamma}}^{\bar{\nu}} A_{\| \bar{v}}^{\alpha}-\Gamma_{\gamma \bar{\delta}}^{v} A_{\| v}^{\alpha}=\Gamma_{\bar{\delta} \gamma}^{\bar{\nu}}\left(A_{\mid v}^{\alpha}-\Gamma_{\beta \psi}^{\alpha} A^{\beta}\right)-\Gamma_{\gamma \bar{\delta}}^{v}\left(A_{\mid v}^{\alpha}-\Gamma_{\beta v}^{\alpha} A^{\beta}\right) \\
& +\Gamma_{\overline{\delta \gamma}}^{\bar{v}}\left(\Gamma_{\beta \psi}^{\alpha}-\Gamma_{\beta \bar{v}}^{\alpha}\right) A^{\beta}-\Gamma_{\gamma \bar{\delta}}^{v}\left(\Gamma_{\beta \psi}^{\alpha}-\Gamma_{\beta \nu}^{\alpha}\right) A^{\beta} \\
& \Gamma_{\overline{\delta \gamma}}^{\bar{V}} A_{\| \bar{\nu}}^{\alpha}-\Gamma_{\gamma \bar{\delta}}^{\nu} A_{\| v}^{\alpha}=\left(\Gamma_{\bar{\delta} \gamma}^{\bar{v}}-\Gamma_{\gamma \bar{\delta}}^{v}\right) A_{\| \psi}^{\alpha}+\Gamma_{\bar{\delta} \gamma}^{\bar{v}}\left(\Gamma_{\beta \psi}^{\alpha}-\Gamma_{\beta \bar{v}}^{\alpha}\right) A^{\beta}-\Gamma_{\gamma \bar{\delta}}^{v}\left(\Gamma_{\beta \psi}^{\alpha}-\Gamma_{\beta v}^{\alpha}\right) A^{\beta}
\end{aligned}
$$

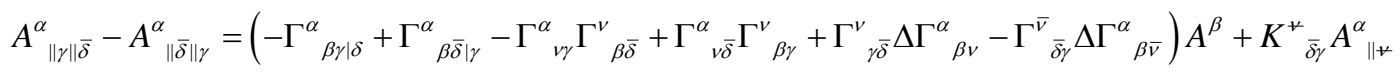

where

$$
\Delta \Gamma_{b c}^{a}=\Gamma_{b c}^{a}-\Gamma_{b c}^{a}=\Gamma_{b c}^{a}-\frac{1}{2}\left(\Gamma_{b c}^{a}+\Gamma_{b \bar{c}}^{a}\right)=\frac{1}{2}\left(\Gamma_{b c}^{a}-\Gamma_{b \bar{c}}^{a}\right)=S_{b c}^{a}
$$

Since the left side and the last term in Equation (5.e) are obviously tensors, then so is the first term which multiples $A^{\beta}$. Then since $A^{\beta}$ is an arbitrary differentiable vector field, its coefficient (the Riemann curvature tensor) must also be a tensor. Using the simplification in Equation (5.3e), we summarize the final result that applies to all eight possible barred and unbarred index combinations in Equations (5.4a) and (5.4b), which use the roman indices that can be barred or unbarred-all combinations giving the correct result. The slashed indices are simply averages of barred and unbarred indices and do not add any more degrees of freedom. Note that indices $a$ and $b$ must have the same type, and that the two internal $m$ and $n$ indices must have types consistent with the types of $c$ and $d$ respectively.

$$
\begin{gathered}
A_{\| c|d| d}^{a}-A^{a}{ }_{\|d\| c}=R_{b c d}^{a} A^{b}+K^{m}{ }_{c d} A^{a}{ }_{\| m m} \\
R_{b c d}^{a}=-\Gamma_{b c \mid d}^{a}+\Gamma_{b d \mid c}^{a}-\Gamma^{a}{ }_{e c} \Gamma_{b d}^{e}+\Gamma_{e d}^{a}{ }_{e d} \Gamma_{b c}^{e}+\left(\Gamma^{m}{ }_{c d} S_{b m}^{a}-\Gamma_{d c}^{n} S_{b n}^{a}\right)
\end{gathered}
$$

We also note a second alternative form of the curvature tensor in Equation (5.4c) which uses covariant derivatives rather than partial derivatives. It is easily verified by expanding the covariant derivative, and is sometimes more useful in derivations because it immediately gives covariant derivatives. It also allows the Riemann curvature to be written with all indices lowered without much change in the form as shown in Equation (5.4d) and then expanded out with simple derivatives in Equation (5.4e). This same technique works with the classical Riemann curvature tensor as well.

$$
\begin{aligned}
& R_{b c d}^{a}=-\Gamma_{b c \mid d}^{a}+\Gamma_{b d \| c}^{a}+\Gamma_{e c}^{a} \Gamma_{b d}^{e}-\Gamma_{e d}^{a} \Gamma_{b c}^{e}+\left(\Gamma_{c d}^{m} S_{b m}^{a}-\Gamma_{d c}^{n} S_{b n}^{a}\right) \\
& R_{a b c d}=-\Gamma_{a b c \| d}+\Gamma_{a b d \| c}+\Gamma_{a e c} \Gamma_{b d}^{e}-\Gamma_{a e d} \Gamma_{b c}^{e}+\left(\Gamma_{c d}^{m} S_{a b m}-\Gamma_{d c}^{n} S_{a b n}\right) \\
& R_{a b c d}=-\Gamma_{a b c \mid d}+\Gamma_{a b d \mid c}-\Gamma_{e b c} \Gamma_{a d}^{e}+\Gamma_{e b d} \Gamma_{a c}^{e}+\left(\Gamma^{m}{ }_{c d} S_{a b m}-\Gamma_{d c}^{n} S_{a b n}\right)
\end{aligned}
$$

One immediate question that arises is how many independent curvature tensors do we have? Since indices a, c and $d$ can be barred or unbarred, we begin with 8. (The type of index $b$ depends on the type of index a and thus is not an independent degree of freedom.) Complex conjugation switches barred and unbarred types and thus divides the number of degrees of freedom in half to 4. Finally, if the last indices are of different type, switching their order simply changes the overall sign of the tensor. This reduces the number of different curvature tensors to 3 .

Curvature Tensors for Lowered Indices: As customary, lowered indices also have their own mixed derivative relationships, which use the same curvature tensors as defined in (5.4). To get the lowered curvature tensors, we simply twice differentiate the scalar function $\Phi=B^{a} A_{a}$, using Equation (4.1c), where $A$ and $B$ are arbitrary twice differentiable vector fields as shown in Equation (5.5a).

$$
\left(B^{a} A_{a}\right)_{\| c|| d}-\left(B^{a} A_{a}\right)_{\|d\| c}=\left(\Gamma_{c d}^{m}-\Gamma_{d c}^{m}\right)\left(B^{a} A_{a}\right)_{\| m}
$$

After some algebra, we get the familiar result in Equation (5.5d) with the expected additional term using the scalar curvature tensor.

$$
\begin{aligned}
& \left(B^{a}{ }_{\|c\| d} A_{a}+B^{a}{ }_{\| c} A_{a \| d}+B^{a}{ }_{\| d} A_{a \| c}+B^{a} A_{a \| c \mid l d}\right)-\left(B^{a}{ }_{\|d\| c} A_{a}+B^{a}{ }_{\| d} A_{a \| c}+B^{a}{ }_{\| c} A_{a \| d}+B^{a} A_{a\|d\| c}\right) \\
& =\left(B^{a}{ }_{\|c \mid\| d}-B^{a}{ }_{\|d\| c}\right) A_{a}+B^{a}\left(A_{a \| c|c| d}-A_{a\|d\| c}\right)=K^{m}{ }_{c d}\left(B^{a} A_{a}\right)_{\|\| m}
\end{aligned}
$$


Using the definition of the Riemann curvature above in Equation (5.3a) and reordering terms, we get Equation (5.5c).

$$
\begin{aligned}
B^{a}\left(A_{a\|c\| d}-A_{a\|d\| c}\right) & =-R_{a c d}^{b} B^{a} A_{b}-K^{m}{ }_{c d} B^{a}{ }_{\| m}+K^{m}{ }_{c d}\left(B^{a} A_{a}\right)_{\| m} \\
& =-R_{a c d}^{b} A_{b} B^{a}+K^{m}{ }_{c d} A_{a \| m} B^{a}
\end{aligned}
$$

Since $B^{a}$ is an arbitrary twice differentiable field, we can remove it from the equality to get Equation (5.4d). This equation is identical to the classical equation except for the addition of the scalar curvature tensor term $K$.

$$
A_{a \| c|| d}-A_{a\|d\| c}=-R_{a c d}^{b} A_{b}+K^{m}{ }_{c d} A_{a \| m}
$$

Thus we see that the curvature tensor for lowered indices is the same curvature tensor as for raised indices with a switch in sign—-the familiar classical result.

\section{Symmetries of the Riemann Curvature Tensor}

Now we get to the critical discussion of the symmetries on the Riemann curvature tensor which will allow us to construct the Einstein tensor and field equations. Symmetries come in two versions. One version has the types moving with the indices, and the other version has types remaining in their fixed position with the indices moving. In classical relativity, all indices are the same type, so the difference never appears.

Last two indices: The most obvious symmetry for the Riemann curvature tensor is the antisymmetry in the last two indices, which trivially comes from the definition of the curvature tensor. This symmetry requires that types move with indices. Thus this symmetry is expressed simply as Equation (6.1).

$$
R_{a b c d}=-R_{a b d c} \text { (typed indices) }
$$

First two indices: We can derive another symmetry relationship for the complex Riemann tensor using techniques similar to ABS. Begin with the scalar function $\phi$ defined as the dot product between two arbitrary twice differentiable vector fields $A^{v}$ and $B^{\tau}$ in Equation (6.2a), and then take the difference of double derivatives as shown in Equations (6.2b). For a scalar function this difference is zero, but it can also be calculated using the derivatives of the vectors as shown in Equation (6.2c). Using Equations (5.3a) and e to write the vector differences in terms of the curvature tensor we get Equation (6.2d).

$$
\begin{gathered}
\phi=A^{v} c_{\nu \bar{\tau}} B^{\bar{\tau}} \\
\phi_{\|\alpha\| \beta}-\phi_{\|\beta\| \alpha}=0 \\
=\left(A_{\|\alpha\| \beta}^{v}-A_{\|\beta\| \alpha}^{v}\right) c_{v \bar{\tau}} B^{\bar{\tau}}+A^{v} c_{v \bar{\tau}}\left(B_{\|\alpha\| \beta}^{\bar{\tau}}-B^{\bar{\tau}}{ }_{\|\beta\| \alpha}\right) \\
=R_{\rho \alpha \beta}^{v} A^{\rho} c_{v \bar{\tau}} B^{\bar{\tau}}+A^{v} c_{v \bar{\tau}} R_{\bar{\rho} \alpha \beta}^{\bar{\tau}} B^{\bar{\rho}}
\end{gathered}
$$

Shuffling some indices and combining terms, we get the Equation (6.3a), which becomes the familiar antisymmetric relationship in Equation (6.3b) when we realize that $A^{v}$ and $B^{\underline{\tau}}$ are arbitrary vector fields.

$$
\begin{gathered}
\left(R_{\bar{\tau} v \alpha \beta}+R_{\bar{v} \alpha \beta}\right) A^{v} B^{\bar{\tau}}=0 \\
R_{\bar{\tau} v \alpha \beta}=-R_{v \bar{\tau} \alpha \beta}
\end{gathered}
$$

The exact same logic holds when $\alpha$ and $\beta$ are both barred indices resulting in Equation (6.3c).

$$
R_{\bar{\tau} v \bar{\alpha} \bar{\beta}}=-R_{v \overline{\tau \alpha} \bar{\beta}}
$$

We also see that the same symmetry holds with mixed type derivatives. We define the scalar function $\phi$ again in Equation (6.2a), and then apply the mixed derivative formula for $\phi$, using Equation (4.1c), to give Equation (6.2b).

$$
\begin{gathered}
\phi=A^{v} c_{v \bar{\tau}} B^{\bar{\tau}} \\
\phi_{\|\alpha\| \bar{\beta}}-\phi_{\|\bar{\beta}\| \alpha}=K_{\alpha \bar{\beta}}^{\rho} \Phi_{\| \rho}=K_{\alpha \bar{\beta}}^{\rho}\left(A^{v} c_{v \bar{\tau}} B^{\bar{\tau}}\right)_{\| \rho}=K_{\alpha \bar{\beta}}^{\rho} A_{\| \rho}^{v} c_{v \bar{\tau}} B^{\bar{\tau}}+A^{v} c_{v \bar{\tau}} K_{\alpha \bar{\beta}}^{\rho} B_{\| \rho}^{\bar{\tau}}
\end{gathered}
$$


Next we expand $\phi$ and differentiate the function piece by piece using the curvature tensor formula in Equation (5.4d) to give Equation (6.2c). Note that all first derivative terms simply subtract away trivially, and that all covariant derivatives of the metric tensor $c_{v \bar{\tau}}$ vanish automatically.

$$
\begin{aligned}
& =\left(A_{\|\alpha\| \bar{\beta}}^{v}-A_{\|\bar{\beta}\| \alpha}^{v}\right) c_{v \bar{\tau}} B^{\bar{\tau}}+A^{v} c_{v \bar{\tau}}\left(B_{\|\alpha\| \bar{\beta}}^{\bar{\tau}}-B_{\|\bar{\beta}\| \alpha}^{\bar{\tau}}\right) \\
& =R_{\rho \alpha \bar{\beta}}^{v} A^{\rho} c_{v \bar{\tau}} B^{\bar{\tau}}+A^{v} c_{v \bar{\tau}} R_{\bar{\rho} \alpha \bar{\beta}}^{\bar{\tau}} B^{\bar{\rho}}+K_{\alpha \bar{\beta}}^{\rho} A_{\| \rho}^{v} c_{v \bar{\tau}} B^{\bar{\tau}}+K_{\alpha \bar{\beta}}^{\rho} A^{v} c_{v \bar{\tau}} B_{\| \rho}^{\bar{\tau}}
\end{aligned}
$$

Realizing that the $K$ terms subtract away and then switching index names to combine terms, we get the Equation (6.5a), which becomes the familiar antisymmetric symmetry relationship in Equation (6.5b) when we realize that $A^{v}$ and $B^{\underline{\tau}}$ are arbitrary vector fields.

$$
\begin{gathered}
\left(R_{\bar{\tau} v \alpha \beta}+R_{v \bar{\tau} \alpha \beta}\right) A^{v} B^{\bar{\tau}}=0 \\
R_{\bar{\tau} v \alpha \beta}=-R_{\bar{v} \bar{\tau} \alpha \bar{\beta}}
\end{gathered}
$$

The final summarized result is that the antisymmetry in the first two indices holds for all possible combinations of barred and unbarred indices as stated in Equation (6.6a).

$$
R_{a b c d}=-R_{b a c d} \quad \text { (typed indices) }
$$

Three-fold symmetry: Another immediately obvious symmetry is a familiar three-fold symmetry but (caution!) here the types do not move with the indices. Such symmetries have not been found to be very useful compared to symmetries where the types move with the indices, but we list it here for completeness. This symmetry follows immediately from inspection of the expressions for the curvature tensor in (5.3c). Since every Christoffel symbol is symmetric in the last two indices, every term immediately zeroes out under the signed sum of permutations. To differentiate clearly between the cases where the types are fixed to positions and where the types are fixed to the indices, we annotate each equation and also use the equation format $\{\cdots\}_{(a, b, c)}$ for typed positions and $\{\cdots\}_{[a, b, c]}$ for typed indices.

$$
\begin{array}{cc}
\left\{R_{b c d}^{a}\right\}_{(b, c, d)}=0 & \text { (typed positions) } \\
\left\{R_{a b c d}\right\}_{(b, c, d)}=0 & \text { (typed positions) } \\
\left\{R_{b a c d}\right\}_{(b, c, d)}=0 & \text { (typed positions) }
\end{array}
$$

Bianchi Identities: From here on, all the symmetries will use typed indices. The Bianchi identities can now be gotten using the following straightforward technique. We simply begin in Equation (6.8a) and (6.8b) with two trivial identities, where the first term of each equation produces the same 6 terms as the second but with opposite signs since two of the indices are switched.

$$
\begin{aligned}
& \left\{A_{a\|b\| c|c| d}\right\}_{[b, c, d]}+\left\{A_{a\|b\| d \| \mid c}\right\}_{[b, c, d]}=0 \text { (typed indices) } \\
& \left\{A_{a\|b\| d \| \mid c}\right\}_{[b, c, d]}+\left\{A_{a \mid d\|b\| c}\right\}_{[b, c, d]}=0 \text { (typed indices) }
\end{aligned}
$$

Subtracting Equation (6.8b) from Equation (6.8a), we get Equation (6.8c). This becomes Equation (6.8d) when we use the Riemann curvature Equation (5.4d).

$$
\begin{aligned}
& \left\{A_{a|b| c|c| d}-A_{a \| b|| d \mid c}\right\}_{[b, c, d]}+\left\{A_{a\|b|d \|| c}-A_{a\|d\| \| b \mid c}\right\}_{[b, c, d]}=0 \text { (typed indices) } \\
& \left\{-R_{a c d}^{n} A_{n \| b}-R_{b c d}^{n} A_{a \| n}+K^{n}{ }_{c d} A_{a\|b\| \sharp}\right\}_{[b, c, d]}+\left\{\left(-R_{a b d}^{n} A_{n}+K_{b d}^{n} A_{a \| \sharp n}\right)_{\| c}\right\}_{[b, c, d]}=0 \text { (typed indices) }
\end{aligned}
$$

Now we expand the $\| c$ derivative in the second term in Equation (6.8e), and switch $b$ and $c$ in the term $K_{c d}^{n} A_{a\|b\| \#}$ along with a sign switch (OK since we have the antisymmetrized [b,c,d] sum applied). 


$$
\begin{aligned}
& \left\{-R^{n}{ }_{a c d} A_{n \| b}-R_{b c d}^{n} A_{a \| n}-K^{n}{ }_{b d} A_{a\|c \mid\| \#}\right\}_{[b, c, d]} \\
& +\left\{-R_{a b d \| c}^{n} A_{n}-R_{a b d}^{n} A_{n \| c}+K^{n}{ }_{b d \| c} A_{a \| \#}+K^{n}{ }_{b d} A_{a\|\#\| c}\right\}_{[b, c, d]}=0
\end{aligned}
$$

We note that the first term in the first line subtracts out the second term in the second line using the antisymmetric sum $[b, c, d]$. We also note that the two double derivative terms combine to give another term with a curvature tensor times $A_{n}$. Doing these simplifications and collecting terms, we have Equation (6.8f).

$$
\left\{R_{b c d}^{n}\right\}_{[b, c, d]} A_{a \| n}+\left\{R_{a b d \| c}^{n}+K_{b d}^{m} R_{a c m}^{n}\right\}_{[b, c, d]} A_{n}-\left\{K_{b d \| c}^{n}+K_{b d}^{m} K_{c m}^{n}\right\}_{[b, c, d]} A_{a \| n}=0
$$

Note that if indices $b, c$ and $d$ were all the same type, then all the $K$ terms would vanish and we would have only two terms left as shown in Equation (6.8g).

$$
\text { If }(b, c, d) \text { have the same type } \rightarrow\left\{R_{b c d}^{n}\right\}_{[b, c, d]} A_{a \| n}+\left\{R_{a b d \| c}^{n}\right\}_{[b, c, d]} A_{n}=0
$$

Since an arbitrary field and its covariant derivative are two independent degrees of freedom, both components must separately vanish to give the familiar Bianchi identities as the coefficient of $A_{n}$ and the familiar three-index symmetry of the Riemann curvature tensor as the coefficient of $A_{a \| n}$. Thus the only issue here is how to extend these results to include indices of different types, and the main issue is how to deal with the $A_{a \| n}$ and the $A_{a \| n}$ terms. Are they independent degrees of freedom or not? We believe that they have the effect of being independent degrees of freedom, which means that all the terms in Equation (6.8f) which multiply either $A_{a \| n}, A_{n}$ or $A_{a \| \uparrow}$ are each individually zero.

The logic supporting this principle goes as follows: The first insight is to point out that the partial derivative $A_{a \mid n}$ is equally present in both the $A_{a \| n}$ and the $A_{a \| n}$ terms and can take on any value independent of $A_{a}$. This means that the sum of those two terms must vanish and also that the middle term multiplying the $A_{n}$ field must independently vanish since $A_{n}$ is an independent degree of freedom. This is enough to prove the generalized Bianchi identities in Equation (6.9a) and the vanishing of the sum in Equation (6.9b).

$$
\begin{aligned}
& \text { Bianchi Identities: }\left\{R_{a b d \| c}^{n}+K^{m}{ }_{b d} R^{n}{ }_{a c m}\right\}_{[b, c, d]}=0 \\
& \text { Triplet Symmetry: }\left\{R_{b c d}^{n}\right\}_{[b, c, d]}-\left\{K^{n}{ }_{b d \| c}+K^{m}{ }_{b d} K^{n}{ }_{c m}\right\}_{[b, c, d]}=0
\end{aligned}
$$

Dropping the now proven zero middle term in Equation (6.8f), we can rewrite Equation (6.8f) as (6.9c). Then using Equation (6.9b), the terms multiplying the derivatives $A_{a \mid n}$ also drop out as zero to give Equation (6.9d).

$$
\begin{gathered}
\left\{R_{b c d}^{n}\right\}_{[b, c, d]} A_{a \| n}-\left\{K_{b d \| c}^{n}+K_{b d}^{m} K_{c m}^{n}\right\}_{[b, c, d]} A_{a \| n}=0 \\
\left(\left\{R_{b c d}^{n}\right\}_{[b, c, d]} \Gamma_{a n}^{e}-\left\{K_{b d \| c}^{n}+K_{b d}^{m} K_{c m}^{n}\right\}_{[b, c, d]} \Gamma_{a+1}^{e}\right) A_{e}=0
\end{gathered}
$$

We then add and subtract terms in Equation (6.9d) to get Equation (6.9e).

$$
\left(\left\{R_{b c d}^{n}\right\}_{[b, c, d]}-\left\{K_{b d \| c}^{n}+K_{b d}^{m} K_{c m}^{n}\right\}_{[b, c, d]}\right) \Gamma_{a n}^{e} A_{e}+\left(\left\{R_{b c d}^{n}\right\}_{[b, c, d]}\right)\left(\Gamma_{a n}^{e}-\Gamma_{a n}^{e}\right) A_{e}=0
$$

Since we have already proven that the first term is identically zero in Equation (6.9b), so is the second term. Also recognizing the difference of the Christoffel symbols from Equation (5.3e) as $S_{a n}^{e}$, we simplify Equation (6.9e) to Equation (6.9f).

$$
\left(\left\{R_{b c d}^{n}\right\}_{[b, c, d]}\right) S_{a n}^{e} A_{e}=0
$$

Given that $A_{e}$ is an arbitrary field and a is an arbitrary index, as long as the $S$ field is even microscopically non-zero everywhere, then we conclude the very simple result in Equation (6.9g).

$$
\left\{R_{b c d}^{n}\right\}_{[b, c, d]}=0
$$

The final results of this analysis are given in Equations (6.10). 


$$
\begin{aligned}
& \text { Bianchi Identities: }\left\{R_{a b d \| c}^{n}+K_{b d}^{m} R_{a c m}^{n}\right\}_{[b, c, d]}=0 \text { (typed indices) } \\
& \text { Triplet } R \text { Symmetry: }\left\{R_{b c d}^{n}\right\}_{[b, c, d]}=0 \text { (typed indices) } \\
& \text { Triplet } K \text { Symmetry: }\left\{K_{b d \| c}^{n}+K_{b d}^{m} K_{c m}^{n}\right\}_{[b, c, d]}=0 \text { (typed indices) }
\end{aligned}
$$

We can also lower the $\mathrm{n}$ indices to get an equivalent form for the Bianchi and triplet identities assuming that only barred and unbarred indices are used. We are, however, cautious about extending the results to slashed indices $A$, which do not have an obvious ability to be raised and lowered with a metric tensor like the other two.

$$
\begin{aligned}
& \text { Bianchi Identities: }\left\{R_{n a b d \| c}+K_{b d}^{m} R_{n a c m}\right\}_{[b, c, d]}=0 \text { (typed indices) } \\
& \text { Triplet } R \text { Symmetry: }\left\{R_{n b c d}\right\}_{[b, c, d]}=0 \text { (typed indices) }
\end{aligned}
$$

Modified Bianchi Identities: To put these equalities into a slightly different form we can use the Equation (6.10b) (with a lowered first index), which says that the sum over all six signed permutations of the last three indices of $R_{\text {nabc }}$ equals 0 . By keeping one term on the left and putting the other 5 terms with opposite sign on the right, we can rewrite the Bianchi identities in Equations (6.10b) as (6.11a). (Note that the c and d index names have been switched here to make the relationship look simpler.)

Since we are antisymmetrizing with the indices $(b, c, d)$, the first term on the right changes sign when we flip the $\mathrm{cb}$ indices, which means it can be added to the left side-keeping everything equal to zero. Similarly the second and fourth terms on the right simply double, and the third and fifth terms also double. Dividing everything by two, we end up with Equation (6.9b).

$$
\begin{gathered}
\left\{R_{\text {eabc||d }}\right\}_{[b, c, d]}=\left\{R_{\text {eacbl|d }}+R_{e b a c \mid d}+R_{\text {ecba||d }}-R_{\text {ecab||d }}-R_{\text {ebcal|d }}\right\}_{(b, c, d)}=\left\{-K^{m}{ }_{b d} R_{e a c m}\right\}_{[b, c, d]} \\
\left\{R_{e b a c|| d}+R_{e c b a \| d}\right\}_{[b, c, d]}=\left\{-K^{m}{ }_{b d} R_{e a c m}\right\}_{[b, c, d]} \text { (typed indices) }
\end{gathered}
$$

Now we can use the antisymmetric property in the last two typed indices (Equation (6.1)) combined with the antisymmetry in the $[b, c, d]$ indices to make the two terms equal, resulting in Equation (6.12a) or equivalently in Equation (12b) and (12c), using the antisymmetric conditions in the first two and last two indices. This is exactly the same symmetry condition as in classical relativity.

$$
\begin{aligned}
& \left\{R_{e b a c|| d}\right\}_{[b, c, d]}=\left\{-K^{m}{ }_{b d} R_{e a c m}\right\}_{[b, c, d]} \text { (typed indices) } \\
& \left\{R_{e b c a \| d}\right\}_{(b, c, d)}=\left\{K^{m}{ }_{b d} R_{e a c m}\right\}_{[b, c, d]} \text { (typed indices) } \\
& \left\{R_{\text {becal|d }}\right\}_{(b, c, d)}=\left\{-K^{m}{ }_{b d} R_{e a c m}\right\}_{[b, c, d]} \text { (typed indices) }
\end{aligned}
$$

Note that if $[b, c, d]$ indices are all the same type, as we will require to derive the Einstein tensor below, then all the $K$ terms vanish since the lower two indices are always of the same type. In particular, Equation (6.12a) will be the starting point for the derivation of the generalized Einstein tensor.

Einstein Tensor: With this background, we can now show the generalization of the zero divergence condition of the Einstein tensor, which will lay the basis for the field equations. The symmetries required for the field equations apply only to a particular set of barred and unbarred indices, namely $R_{\alpha \bar{\beta} \gamma \bar{\delta}}$. Since we need to be able to contract the tensors in the Einstein field equation, which requires both barred and unbarred indices to be present. First, however, we need to define some contracted tensors for clarity. With two barred and two unbarred indices in $R_{\alpha \bar{\beta} \gamma \bar{\delta}}$, we can contract to a two dimensional tensor in four different ways—all of which are needed to make the zero divergence Einstein tensor.

$$
\begin{gathered}
R_{\bar{\beta} \gamma}=-R_{\alpha \bar{\beta} \gamma \bar{\delta}} c^{\alpha \bar{\delta}} \\
R_{\gamma \bar{\beta}}=-R_{\bar{\gamma} \bar{\alpha} \bar{\beta}} C^{\alpha \bar{\delta}}=-R_{\bar{\delta} \bar{\gamma} \bar{\beta} \alpha} C^{\alpha \bar{\delta}}=R_{\overline{\gamma \beta}}{ }^{*}
\end{gathered}
$$




$$
\begin{aligned}
R_{\beta \bar{\gamma}}^{\prime} & =R_{\alpha \bar{\delta} \beta \bar{\gamma}} C^{\alpha \bar{\delta}}=R_{\bar{\delta} \alpha \bar{\gamma}} C^{\alpha \bar{\delta}}=R_{\gamma \bar{\beta}}^{{ }^{*}} \\
R^{\prime \prime}{ }_{\beta \bar{\gamma}} & =R_{\beta \bar{\gamma} \alpha \bar{\delta}} C^{\alpha \bar{\delta}}=R_{\overline{\gamma \beta} \bar{\delta} \alpha} C^{\alpha \bar{\delta}}=R^{\prime \prime}{ }_{\gamma \bar{\beta}}{ }^{*}
\end{aligned}
$$

Note that two of the double contractions are equal i.e.

$$
R^{\prime}=R^{\prime \prime}
$$

Now to get the generalization of the zero divergence Einstein tensor, begin with Equation (6.15a) (a modified Bianchi identity) and write out all the terms explicitly in Equation (6.15a). Note that the generalized Latin indices have been made specific typed indices here. Also note that here all the permuted terms are the same type, which means that the $K$ term is zero, resulting in a very traditional and familiar result.

$$
\left\{R_{\alpha \bar{\beta} \gamma \bar{\gamma} \| \bar{\eta}}\right\}=R_{\alpha \bar{\gamma} \bar{\gamma} \| \bar{\eta}}-R_{\alpha \bar{\beta} \eta \bar{\eta} \| \bar{\delta}}+R_{\alpha \bar{\eta} \bar{\gamma} \bar{\beta} \| \bar{\delta}}-R_{\alpha \bar{\eta} \bar{\gamma} \bar{\phi} \| \bar{\beta}}+R_{\alpha \bar{\delta} \bar{\gamma} \bar{\eta} \| \bar{\beta}}-R_{\alpha \bar{\delta} \bar{\gamma} \bar{\beta} \| \bar{\eta}}=0
$$

Now contract Equation (6.15a) with $c^{\alpha \underline{\underline{\delta}}}$ and $c^{\gamma \underline{\underline{\eta}}}$ to get (6.15b).

$$
-R_{\bar{\beta} \gamma}{ }^{\| \gamma}-R_{\gamma \bar{\beta}}^{\prime \prime}-R_{\gamma \bar{\beta}}{ }_{\gamma / \gamma}+R_{\| \bar{\beta}}+R^{\prime}{ }_{\| \bar{\beta}}-R_{\gamma \bar{\beta}}^{\prime}{ }_{\gamma / \gamma}
$$

This can be rewritten as follows when we remember that $R^{\prime}=R^{\prime \prime}$.

$$
\left(R_{\bar{\beta} \gamma}+R_{\gamma \bar{\beta}}-c_{\gamma \bar{\beta}} R\right)^{\| / \gamma}+\left(R_{\gamma \bar{\beta}}^{\prime}-\frac{1}{2} c_{\gamma \bar{\beta}} R^{\prime}\right)^{\| \gamma}+\left(R^{\prime \prime}{ }_{\gamma \bar{\beta}}-\frac{1}{2} c_{\gamma \bar{\beta}} R^{\prime \prime}\right)^{\| \gamma}=0
$$

This then will satisfy a zero divergence criterion if we define the generalized Einstein tensor as

$$
2 G_{\bar{\beta} \gamma}=\left(R_{\bar{\beta} \gamma}+R_{\gamma \bar{\beta}}-c_{\gamma \bar{\beta}} R\right)+\left(R^{\prime}{ }_{\gamma \bar{\beta}}-\frac{1}{2} c_{\gamma \bar{\beta}} R^{\prime}\right)^{\| \gamma}+\left(R^{\prime \prime}{ }_{\gamma \bar{\beta}}-\frac{1}{2} c_{\gamma \bar{\beta}} R^{\prime \prime}\right)^{\| \gamma}=0
$$

The generalized Einstein tensor can also be written in the more compact (and = familiar looking) form of Equation (6.16b) where the contracted curvature tensor $R^{s}$ is a sum of the four different contracted forms as shown in Equation (6.16c).

$$
G_{\bar{\beta} \gamma}=R_{\bar{\beta} \gamma}^{s}-\frac{1}{2} c_{\gamma \bar{\beta}} R^{s}
$$

where

$$
2 R_{\bar{\beta} \gamma}^{s}=R_{\bar{\beta} \gamma}+R_{\gamma \bar{\beta}}+R_{\gamma \bar{\beta}}^{\prime}+R_{\gamma \bar{\beta}}^{\prime \prime}
$$

Since the sum $R_{\bar{\beta} \gamma}+R_{\gamma \bar{\beta}}$ is Hermitian from Equations (6.13a) and (6.13b), and since $R_{\gamma \bar{\beta}}^{\prime}$ and $R^{\prime \prime}{ }_{\gamma \bar{\beta}}$ are each Hermitian from Equations (6.13c) and (6.13d), the tensor $R_{\bar{\beta} \gamma}^{s}$ is also Hermitian. Since the metric tensor $c_{\gamma \beta}$ is also Hermitian, the full Einstein tensor is thus Hermitian.

The final result of zero divergence is then summarized in Equation (6.17), which looks exactly like the familiar classical conservation equation except for the bar above the first index.

$$
G_{\bar{\beta} \gamma} \| \gamma=0
$$

Overview of the Field Equations: In classical relativity, the Einstein tensor is set equal to a general stressenergy-momentum tensor that also satisfies the zero divergence condition. This was an ad hoc assumption extrapolated from known gravitational laws at the time, and its solutions have occupied a significant number of scientists for many different scenarios. Equation (6.18) is thus expected to be one of the field equations of the generalized Riemannian geometry. However, it does contain more than gravity. It contains four different types of field: gravity $g_{\mu v}$, electromagnetism $f_{\mu v}$ and two other third rank fields $S_{\mu v \rho}$ and $W_{\mu v \rho}$, which together form mass-energy and charge source terms. Thus this form of Riemannian geometry offers the possibility of a self-contained geometry including all mass-energy and charge sources.

\section{Summary}

In this paper we have explored complex Riemannian geometry further. In particular, we have derived the Riemann curvature tensors (three different ones) and the symmetries that go with them. Using those results, we 
found the generalized Einstein tensor which satisfies the familiar zero divergence condition suitable for field equations. The next paper will propose and explore field equations for all four of the geometric fields, gravity, electromagnetism and two others that provide mass and charge sources.

\section{Conclusion}

We see that complex Riemannian geometry inherently has a metric with a real gravity field and an imaginary part resembling the electromagnetic field plus two wholly new fully symmetric third rank tensor fields $W_{\alpha \beta \gamma}$ and $S_{\alpha \beta \gamma}$ which represent additional potential interactions. The real symmetric fields $W$ and $S$ each have 24 degrees of freedom, and are PT symmetry breaking in that $S$ and $W$ reverse sign when $x^{\alpha} \Rightarrow-x^{\alpha}$. Assuming that CPT will remain a valid symmetry, one might expect that $S$ and $W$ will be involved in charge-current sources so that under all three inversions $S$ and $W$ would remain unchanged. The third and final paper of this series explores the Einstein tensor field Equation (6.18) above plus other field equations required for the electromagnetic field and the two third rank $S$ and $W$ fields. Only more work can tell if this complex Riemannian geometry offers a pathway into a fully unified theory.

\section{References}

[1] Weyl, H. (1918) Gravitation und Elektrizität. Sitzungsberichte der Königlich Preussischen Akademie der Wissenschaften, 465.

[2] Kaluza, T. (1921) Zum Unitätsproblem in der Physik. 2nd Edition, Math. Phys, Berlin, 966-972.

[3] Eddington, A.S. (1924) The Mathematical Theory of Relativity. 2nd Edition, Cambridge University Press, Cambridge.

[4] Mie, G. (1912) Annals of Physics, 37, 511-534. http://dx.doi.org/10.1002/andp.19123420306

[5] Reichenbächer, E. (1917) Annals of Physics, 52, 134-173. http://dx.doi.org/10.1002/andp.19173570203

[6] Hutchin, R.A. (2015) Journal of Modern Physics, 6, 749-757. http://dx.doi.org/10.4236/jmp.2015.66080

[7] Adler, R., Basin, M. and Schiffer, M. (1975) Introduction to General Relativity. McGraw Hill, New York. 